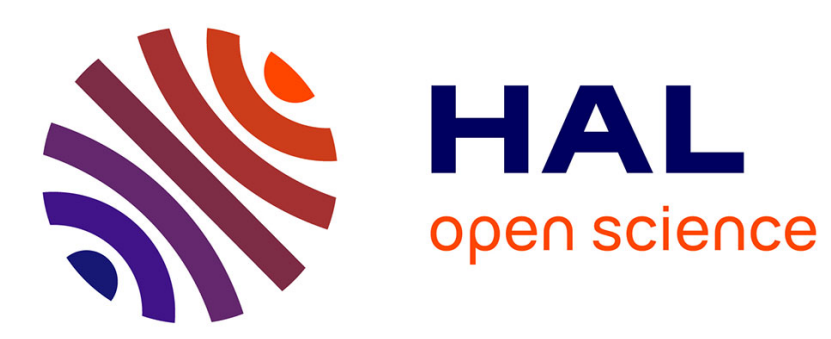

\title{
Single stranded siRNA complexation through non-electrostatic interactions
}

Lucie Giraud, Warren Viricel, Jeanne Leblond, Suzanne Giasson

\section{To cite this version:}

Lucie Giraud, Warren Viricel, Jeanne Leblond, Suzanne Giasson. Single stranded siRNA complexation through non-electrostatic interactions. Biomaterials, 2016, 10.1016/j.biomaterials.2016.10.035 . hal02512428

\section{HAL Id: hal-02512428 \\ https://hal.science/hal-02512428}

Submitted on 23 Mar 2020

HAL is a multi-disciplinary open access archive for the deposit and dissemination of scientific research documents, whether they are published or not. The documents may come from teaching and research institutions in France or abroad, or from public or private research centers.
L'archive ouverte pluridisciplinaire HAL, est destinée au dépôt et à la diffusion de documents scientifiques de niveau recherche, publiés ou non, émanant des établissements d'enseignement et de recherche français ou étrangers, des laboratoires publics ou privés. 


\title{
Single Stranded siRNA Complexation through Non- Electrostatic Interactions
}

\author{
Lucie Giraud $^{1}$, Warren Viricel ${ }^{1}$, Jeanne Leblond ${ }^{1}$ and Suzanne Giasson ${ }^{1,2 *}$ \\ ${ }^{1}$ Faculty of Pharmacy and ${ }^{2}$ Department of Chemistry, Université de Montréal, C.P. 6128, \\ succursale Centre-ville, Montréal, Québec, Canada H3C $3 J 7$
}

*Corresponding author. E-mail: suzanne.giasson@umontreal.ca.

Tel: +1-514-3405175; Fax: +1-514-3405290. 


\begin{abstract}
As double stranded, single stranded siRNA (ss-siRNA) has demonstrated gene silencing activity but still requires efficient carriers to reach its cytoplasmic target. To better understand the fundamental aspect driving the complexation of ss-siRNA with nanocarriers, the interactions between surfaces of various compositions across a ss-siRNA solution were qualitatively investigated using the Surface Forces Apparatus. The results show that ss-siRNA can adsorb onto hydrophilic (positively and negatively charged) as well as on hydrophobic substrates suggesting that the complexation can occur through hydrophobic interactions and hydrogen bonding in addition to electrostatic interactions. Moreover, the binding strength and the conformation of ss-siRNA depend on the nature of the interactions between the ss-siRNA and the surfaces. The binding of ss-siRNA with nanocarriers, such as micelles or liposomes through non-electrostatic interactions was also evidenced by a SYBR ${ }^{\circledR}$ Gold cyanine dye. We evidenced the presence of interactions between the dye and oligonucleotides already complexed to non-cationic nanovectors biasing the quantification of the encapsulation. These results suggest that non-electrostatic interactions could be exploited to complement electrostatic interactions in the design of nanocarriers. In particular, the types of interaction between ss-siRNA and the carriers can be tuned to decrease the cationic charges and therefore lower its toxicity.
\end{abstract}

Keywords: single stranded RNA, surfaces forces apparatus, hydrophobic interactions, hydrogen bonding 


\section{Introduction}

Small interfering ribonucleic acids (siRNA) are small double strand RNA molecules of 20-30 base pairs [1] capable of inhibiting specific gene expression in plants [2] and animals [3] by causing cleavage of specific messenger RNA (mRNA) after transcription. The specific gene silencing activity of double stranded siRNA (ds-siRNA) is currently clinically assessed to treat large variety of diseases, such as genetic disorders,[4] cancer [5] or infectious diseases.[6] Compared to other gene silencing strategies, such as single-stranded antisense RNA or steric-blocking oligonucleotides,[7] siRNA require a lower dosage, since a single molecule can cleave a large number of target mRNA. Interestingly, several studies have shown that only one RNA strand is required to activate the RNAi pathway and that single stranded siRNA (ss-siRNA) can bypass the ds-siRNA form.[8, 9] Lima et al. have recently optimized the ss-siRNA structure to silence protein expression with potency similar to the duplex.[10] Moreover, ds-siRNA is known to induce non-specific gene silencing, [11] a phenomenon caused by the saturation of the endogenous RNAi machinery by sense and antisense siRNA strands [12], resulting in toxic phenotypes.[13] Therefore, delivering ss-siRNA prevents from administrating the sense strand of ds-siRNA, which would contribute to reduce the non-specific gene silencing [14] and other toxicity issues.[7, 15, 16]

Because of its high negative charge and hydrophilic nature, siRNA does not cross biological membranes easily, which limits its bioavailability.[17] In some clinical applications, naked siRNA can be administered locally, such as in lung, eyes, and muscles.[18, 19] However, many tissues can only be reached after systemic administration, such as the liver, a major target of siRNA therapeutics in clinics.[18] In physiological conditions, siRNA can be rapidly eliminated by endogenous nucleases degradation,[20] by the reticuloendothelial system [21] or by glomerular filtration. Therefore, many recent efforts have been focused on the careful design of drug delivery systems (DDS), able to overcome each of the biological barriers encountered until the cytoplasm of the cell, i.e. the siRNA's site of action.[17, 18, 21]

Viral vectors are among the first vehicles studied for small oligonucleotides delivery but can induce unacceptable toxicity.[21] The complexation of ds-siRNA with synthetic vectors such as lipid nanoparticles,[22-24] cyclodextrin based polymeric 
nanoparticles $[25,26]$ or polymeric nanoparticles $[27,28]$ have been extensively studied and some of them are currently under clinical trials. $[18,29]$ Whatever the nanocarrier, they are generally positively charged and involve complexation of the negatively charged siRNA through electrostatic interactions.[23, 30, 31] However, this cationic feature has been related to higher toxicity compared to uncharged or anionic carriers. Such toxicity is associated with non-specific interactions and internalization with cells, a high rate of opsonisation and a short blood circulation half-life,.[32, 33] To optimize the nanocarrier design, extensive chemical diversity has been explored. High throughput screening of cationic lipids, for instance, has shown that too many or too strong (e.g. quaternary amines) cationic groups can significantly impede the cytosolic release of the siRNA payload, and limit the transfection efficiency.[34] Interestingly, several studies have demonstrated that ds-siRNA and ss-mRNA do not require the same carrier for optimized delivery.[35] However, structure-activity relationships are not deeply understood, and more in-depth knowledge about siRNA and the nanocarrier interactions would be a great advantage for the design of efficient and safe DDS.

Theoretical studies have shown that double and single stranded oligonucleotides can interact with hydrophilic and hydrophobic surfaces through different kind of interactions.[36-39] However, experimental investigation of single stranded oligonucleotides complexation with surfaces and more particularly with DDS has been less reported. Because of the short sequence, 3D structure of ss-siRNA is quite simple or inexistent [40] and most probably lost during adsorption onto the surface. Nevertheless, thanks to its variety of chemical groups, ss-siRNA can be a partner for hydrogen bonding, hydrophobic and/or electrostatic interactions, as illustrated in Figure S1. Hydrophobic interactions are possible [41] and probably via the aromatic cycle, hydrogen bonding via the hydroxyl carried by ribose or amino groups of nucleobases and electrostatic interactions via the negatively charged phosphate groups of ribose or positively charged amino groups of the nucleobases. In this study, the binding mechanisms of ss-siRNA with carriers was investigated by measuring the adsorption of ss-siRNA onto hydrophilic (positively and negatively charged) and hydrophobic surfaces using the Surface Forces Apparatus (SFA). The ability of charged and uncharged DDS to encapsulate ss-siRNA using electrostatic and 
non-electrostatic interactions was then demonstrated with micelles and liposomes of various chemical nature and surface charges using fluorescence assays.

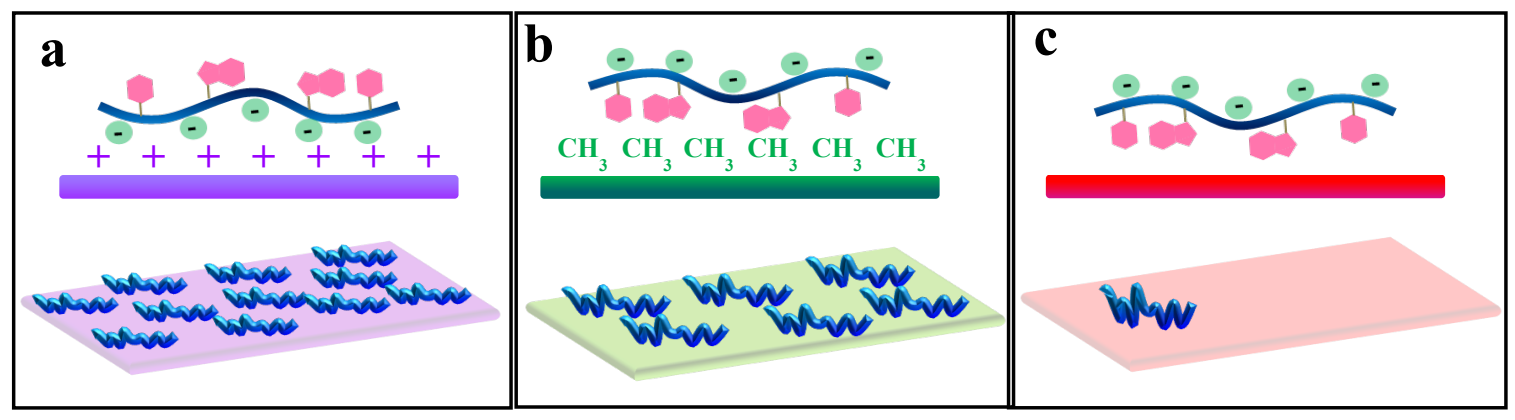

Figure 1: Schematic representations of the conformation of adsorbed ss-siRNA on (a) aminofunctionalized mica, (b) hydrophobized mica and (c) bare mica deduced from surface forces measurements and AFM images.

\section{Materials and Methods}

Ruby mica sheets were purchased from S \& J Trading, Inc. (Glen Oaks, NY, USA). Plasma Prep II from SPI Supplies was used to activate freshly cleaved mica surfaces using argon (5.0 grade). Milli-Q quality water was obtained from a Millipore Gradient aminoundecyltriethoxysilane (AUTES) was purchased from Gelest, Inc, (Morrisville, PA, USA). Dodecyltrichlorosilane was purchased from Sigma-Aldrich (Oakville, ON, Canada). Single strand siRNA (5'-UAAGGCUAUGAAGAGAUAC-3') was purchased from Fidelity Systems, Inc., (Gaithersburg, MD, USA). Cholesterol (Chol), 1-2-dioleoylsn-glycerol (DAG), 1,2-dioleoyl-3-trimethylammonium-propane (chloride salt) (DOTAP) and 1,2-dimyristoyl-sn-glycero-3-phospho-(1-rac-glycerol) (sodium salt) (DMPG) were purchased from Avanti Polar Lipids (Alabaster, AL, USA). Poly(styrene-block-N-methyl4-vinyl pyridinium iodide, $\left(\mathrm{PS}_{3.5}\right.$-b-PM4VPI 11.6$)$, poly(styrene-block-acrylic acid) $\left(\mathrm{PS}_{3.2}\right.$ b-PAA $\left.{ }_{12.5}\right)$ and poly(styrene-block-ethylene glycol) $\left(\mathrm{PS}_{3.2}-\mathrm{b}-\mathrm{PEG}_{12.5}\right)$, the indexed values correspond to the molecular weight in $\mathrm{kDa}$, were purchased from Polymer Source Inc. (Montreal, QC, Canada). SYBR ${ }^{\circledR}$ Gold fluorescent cyanine dye was purchased from Thermo Fisher Scientific (Ottawa, ON, Canada).

\subsection{Liposome preparation}

Stock solutions (10-20 mg/mL) of commercial lipids and cholesterol were prepared in chloroform. The lipid stock solutions were combined in a $5 \mathrm{~mL}$ round-bottom flask at a $50 / 50$ molar ratio ( $5 \mu \mathrm{mol}$ of lipid and $5 \mu \mathrm{mol}$ of cholesterol). The chloroform was removed under reduced pressure and the lipid film was further dried for 1 hour under high vacuum 
to remove any residual solvent. The dried lipid film thus obtained was hydrated with $1 \mathrm{~mL}$ of Milli-Q water. This solution was heated to $65^{\circ} \mathrm{C}$ and vortexed 2 minutes to obtain a homogenous suspension of large multilamellar liposomes. The solution was then extruded 11 times through a $200 \mathrm{~nm}$ polycarbonate membrane using a LiposoFast manual extruder (Avestin Inc., Ottawa, ON, Canada). Liposome preparations were stored in darkness at $4{ }^{\circ} \mathrm{C}$ in microcentrifuge tubes. Cholesterol, DOTAP, DMPG and DAG successfully incorporated in the liposomal formulations were quantified by HPLC-UV/MS using an Agilent 1260 Infinity HPLC equipped with a UV detector and a 6120 single-quad mass spectrometer (Mississauga, ON, Canada). Detailed procedures are summarized in Table $\mathrm{S} 1$.

\subsection{Micelle preparation}

Cationic, anionic and uncharged micelles were prepared in water by direct dissolution using $\mathrm{PS}_{3.5}-\mathrm{b}-\mathrm{PM} 4 \mathrm{VPI}_{11.6}, \mathrm{PS}_{3.2}-\mathrm{b}-\mathrm{PAA}_{12.5}$ ) and $\mathrm{PS}_{3.2}-\mathrm{b}-\mathrm{PEG}_{12.5}$, respectively. Block copolymers were dissolved in Milli-Q water $(0.2 \mathrm{~g} / \mathrm{L})$ at $\mathrm{pH} 10$ for $\mathrm{PS}_{3.2}-\mathrm{b}-\mathrm{PAA}_{12.5}$ and $\mathrm{pH}$ 5.8 for $\mathrm{PS}_{3.5}-\mathrm{b}-\mathrm{PM}_{4} \mathrm{VPI}_{11.6}$ and $\mathrm{PS}_{3.2}-\mathrm{b}-\mathrm{PEG}_{12.5}$. The resulting micellar suspensions were adjusted to $\mathrm{pH} 5.8$ when required and sonicated for $1 \mathrm{~h}$ prior to use.

\subsection{DDS characterization}

The hydrodynamic diameter and zeta potential of suspended liposomes and micelles in Milli-Q water before and after co-incubation with ss-siRNA were determined using a dynamic light scattering (DLS, Zetasizer Nano ZS, Malvern Instrument Ltd., Malvern, Worcestershire, UK) at $20^{\circ} \mathrm{C}$ in back-scattering mode. Mean values of triplicates are reported in the result section.

\section{4 sS-siRNA complexation with DDS and encapsulation efficiency}

A SYBR ${ }^{\circledR}$ Gold assay was used to quantify the ss-siRNA encapsulation efficiency of the formulations.[42-44] Stock solutions of DDS (liposomes or micelles) and ss-siRNA were diluted in Milli-Q water at appropriate concentrations, for given lipid/ss-siRNA or copolymer/ss-siRNA ratio. DDS suspensions and ss-siRNA solutions were all prepared for a final ss-siRNA concentration of $250 \mathrm{nM}$. ss-siRNA solutions were added to the DDS suspension followed by brief vortexing. Complexation was allowed to occur for 30 minutes at room temperature. After complexation, the solutions were centrifuged at 20,000 $\mathrm{g}$ for 30 minutes. ss-siRNA in the supernatant was quantified against a calibration curve of ss- 
siRNA (20-250 nM), using an excess of $\mathrm{SYBR}^{\circledR}$ Gold fluorescent cyanine dye $\left(\lambda_{\text {ex/em }}=\right.$ 495/537 nm) and a Safire microplate reader (Tecan, Seestrasse, Switzerland). ss-siRNA encapsulation efficiency (\%) was calculated assuming that measured fluorescence intensity at $537 \mathrm{~nm}$ corresponds to the unencapsulated ss-siRNA as follow:

$$
E E(\%)=\frac{250 \mathrm{nM}-\text { ss. siRNA in the mixture after centrifugation }(\mathrm{nM})}{250 \mathrm{nM}}
$$

The fluorescence emission spectra of SYBR ${ }^{\circledR}$ Gold (cyanine) intercaling ss-siNRA with and without DDS were recorded in Safire microplate reader.

The anisotropy of emission fluorescence of SYBR ${ }^{\circledR}$ Gold (cyanine) intercaling sssiRNA with and without DDS were acquired using a Cary Eclipse fluorescence spectrophotometer (Varian) and a manual polarizer accessory with an excitation wavelength of $495 \mathrm{~nm}$ in Milli-Q water at $20^{\circ} \mathrm{C}$. The fluorescence anisotropy $(\mathrm{r})$ is defined according to the following equation:

$$
r=\frac{I_{\|}-I_{\perp}}{I_{\|}-2 I_{\perp}}=\frac{I_{V V}-G \cdot I_{V H}}{I_{V V}+2 G \cdot I_{V H}}
$$

where $I_{\|}$and $I_{\perp}$ are the parallel and perpendicular fluorescence and can be access by measuring the $\mathrm{IVv}_{\mathrm{V}}$ and $\mathrm{I}_{\mathrm{VH}}$ are the fluorescence intensities obtained using vertically polarized excitation light and recording the horizontal $\left(\mathrm{I}_{\mathrm{VH}}\right)$ or the vertical ( $\mathrm{IVv}_{\mathrm{Vv}}$ fluorescence polarized emission, respectively. The $\mathrm{G}$ factor is an instrumental parameter that corrects polarization effects introduced by the fluorescence detecting system and is defined

as:

$$
G=\frac{I_{H V}}{I_{H H}}
$$

where $\mathrm{I}_{\mathrm{HV}}$ and $\mathrm{I}_{\mathrm{HH}}$ are the fluorescence intensities, obtained using horizontal polarized exciting light and recording the vertical $\left(\mathrm{I}_{\mathrm{HV}}\right)$ and horizontal $\left(\mathrm{I}_{\mathrm{HH}}\right)$ fluorescence polarized emission, respectively.

\subsection{Surface preparation}

Bare mica, amino-functionalized and hydrophobized mica surfaces were used as model for negatively charged, positively charged and hydrophobic surfaces respectively. All surface manipulations were performed in a clean laminar airflow cabinet in order to prevent dust deposition on the surfaces. All glassware was carefully cleaned in a bath of 
$\mathrm{KOH}$-saturated isopropyl alcohol and then rinsed intensively with Milli-Q water and ethanol.

Surface activation of mica substrates: Water/argon plasma activation was used to generate silanol groups on the mica surfaces as previously reported.[45] Briefly, freshly cleaved mica surfaces were placed in a plasma chamber under a vacuum pressure of $0.5 \mathrm{mTorr}$. Argon and water vapor were then introduced at partial pressures of $80 \mathrm{mTorr}$ and 300 mTorr respectively. Plasma activation was performed for $5 \mathrm{~min}$ at $40 \mathrm{~W}$ then, mica surfaces were left in the plasma chamber under vacuum $(0.5 \mathrm{mTorr})$ for $5 \mathrm{~min}$. The mica surfaces were removed from the chamber and immediately used for grafting procedures. Amino-functionalization of mica substrates: AUTES solution of $1 \mathrm{mM}$ was prepared in absolute ethanol and stirred for 30 minutes. Immediately after this step, activated mica substrates were immersed into the AUTES solution for $20 \mathrm{~min}$. The resulting AUTESfunctionalized surfaces were thoroughly rinsed with anhydrous ethanol. The AUTES grafting was completed by annealing the substrates for $30 \mathrm{~min}$ at $120^{\circ} \mathrm{C}$ under atmospheric pressure.

Hydrophobization of mica substrates: Hydrophobic monolayers made of dodecyltrichlorosilane were grafted to activated mica substrates. A solution of $100 \mathrm{mM}$ of dodecyltrichlorosilane in dry toluene was stirred $1 \mathrm{~h}$ then filtered on $0.2 \mu \mathrm{m}$ polytetrafluoroethylene (PTFE) filter. Immediately after the activation, the substrates were immersed in the dodecyltrichlorosilane solution under inert atmosphere. After $2 \mathrm{~h}$, the substrates were removed and rinsed with dry toluene, dried with nitrogen and annealed for $1 \mathrm{~h}$ in $120^{\circ} \mathrm{C}$ under atmospheric pressure.

ss-siRNA adsorption on substrate: A drop of ss-siRNA solution (5 $\mu \mathrm{M}$ in Milli-Q water, filtered with a sterile Anotop 10 syringe filter or $0.02 \mu \mathrm{m}$ ) was deposited on the substrates and covered with a glass slide to spread it. After $2 \mathrm{~h}$ of adsorption, the surfaces were gently washed with Milli-Q water and dried with nitrogen.

\subsection{Surface characterization}

\section{Atomic Force Microscopy (AFM)}

AFM imaging was carried out using a Multimode microscope equipped with a Nanoscope V extend controller (Digital Instruments, Santa Barbara, CA) at room temperature and in ambient air. The surfaces were imaged in the tapping-mode using a silicon cantilevered tip 
from AppNano with a resonance frequency of $200-400 \mathrm{kHz}$ and a spring constant between 25 and $75 \mathrm{~N} / \mathrm{m}$. AFM images were treated and analyzed using Nanoscope Analysis (version 1.4) software. All the AFM topography images presented in the result section are representative of several areas of a given substrate. The roughness of the surfaces was determined and averaged by extracting the root mean square (RMS) on a minimum of three different areas of various scan size.

\section{Contact angle measurement}

Static water contact angle on the different functionalized substrates were measured using a FTA200 dynamic contact angle analyzer (First Ten Angstrom). All measurements were carried out in the static mode. A droplet of Milli-Q water (3-5 $\mu \mathrm{L})$ was deposited on the surfaces and a clear picture of it was taken and used to determine the contact angle within an error of $\pm 0.6^{\circ}$. Fta32 Video software was used for data analyses. The contact angles were measured on three different areas of a given substrate and the mean value is reported in the result section. The water contact angle measured on freshly activated mica and silica substrates is $0^{\circ}$ (Table 1). An increase in water contact angle is expected to occur after the immobilization of alkylsilane owing to the hydrophobic character of the grafted alkyl chain.[46]

\section{Zeta potential measurements on planar surfaces}

Zeta potential on flat bare mica and AUTES-functionalized mica substrates (produced from $1 \mathrm{mM}$ and an immersion time of $20 \mathrm{~min}$ ) were done using Zetasizer Nano ZS instrument (Malvern Instrument Ltd., Malvern, Worcestershire, UK) at $25^{\circ} \mathrm{C}$ using the Surface Zeta Potential Cell with an oppositely charged tracer. The $\mathrm{pH}$ of tracer solution was adjusted using $\mathrm{HCl}$.

\section{Force-Distance measurements}

The normal interaction forces between the surfaces were measured as a function of separation distance using a SFA 2000 and a Mark II Surface Forces Apparatus (SFA) at $25^{\circ} \mathrm{C}$. The SFA and detailed experimental procedures for measuring the normal forces are well described elsewhere.[47] In brief, back-silvered molecularly smooth mica surfaces are glued onto two cylindrically curved silica lenses of radius $\mathrm{R}=2 \mathrm{~cm}$. A force-distance profile between the surfaces is obtained by changing the position of the lower surface, using a nanopositioner. An interferometry technique using Fringes of Equal Chromatic Orders 
(FECO) is used to determine the separation distance between the surfaces with subnanometric resolution.[48] The interaction force is then determined from the deflection of the spring using Hooke's law. Prior to all experiments, two bare substrates (mica surfaces) were brought into adhesive contact in air in order to determine the reference position $(\mathrm{D}=0)$. The SFA disks were dismounted under a laminar-flow cabinet when surface modification is required. After the surface functionalization, the disks were mounted back in the SFA chamber for determining the force-distance profiles between the two surfaces. For the force measurement force profiles across water, a droplet of pure water (Milli-Q water) was injected between the surfaces and the system was allowed to equilibrate for 1 hour prior to measurements. After the force measurement, water was replaced by ss-siRNA solution of $5 \mu \mathrm{M}$ in Milli-Q water (filtered with a sterile Anotop 10 syringe filter or $0.02 \mu \mathrm{m}$ ) and the system was allowed to equilibrate for 2 hours. The forcedistance profiles across ss-siRNA solution were measured step-wise during approach and separation of the two surfaces. Each separation distance was measured at equilibrium, which was arbitrarily set for a distance variation of less than $0.3 \mathrm{~nm} / \mathrm{min}$.

The directly measured force $F$ between the cylindrically curved surfaces can be related to the free energy of interaction per unit area between two infinite flat surfaces $E_{\text {Flat }}$ using the Derjaguin approximation:[49]

$$
E_{\text {Flat }}=\frac{F}{2 \pi R}
$$

where $\mathrm{F}$ is the measured force and $\mathrm{R}$ the radius of the surfaces. The electrostatic forces were analyzed using the nonlinear Poisson-Boltzmann equation (PB) using constant surface potential boundary conditions. The Debye length was extracted from the exponential decay slope of the force-distance measurement at long separation distance and the surface potential was used as variable parameter. To calculate the apparent electrostatic force across the adsorbed ss-siRNA layers, they were considered as homogeneous layers of thickness corresponding to half the separation distance at close contact $\left(D_{c}\right)$. Therefore, the exponential fitting to the long-ranged forces was done assuming that the effective surface potential is at $D_{\mathcal{C}} / 2$ away from each substrate. 


\section{Results and Discussion}

\subsection{Substrate characterization}

The three model surfaces were characterized in order to confirm their difference in surface properties more particularly the hydrophilicity and surface zeta potential. Smooth freshly cleaved mica substrates resulted in purely hydrophilic surface with a water contact angle of $0^{\circ}$ (Table 1, Fig. 2.b). As expected, mica surface was negatively charged in aqueous media at $\mathrm{pH} 7$ with a zeta potential of $-48 \mathrm{mV}$ (Table 1). Mica substrates were then functionalized in order to prepare hydrophobic and positively charged supported

\begin{tabular}{|c|c|c|c|c|}
\hline Surface & $\begin{array}{c}\text { Static water } \\
\text { contact angle } \\
\theta_{\text {water }}\left({ }^{\circ}\right) \\
\end{array}$ & $\begin{array}{c}\text { Surface zeta } \\
\text { potential } \\
\zeta(\mathrm{mV})^{1} \\
\end{array}$ & $\begin{array}{l}\text { Thickness } \\
(\mathbf{n m})^{2}\end{array}$ & $\begin{array}{l}\text { Surface roughness } \\
\text { RMS }(\mathbf{n m})^{3}\end{array}$ \\
\hline Bare mica & 0 & $-48 \pm 2$ & 0 & $0.07 \pm 0.01$ \\
\hline $\begin{array}{l}\text { Amino- } \\
\text { functionalized mica }\end{array}$ & $78 \pm 2$ & $+40 \pm 4$ & $1.5 \pm 0.2$ & $0.08 \pm 0.01$ \\
\hline Hydrophobized mica & $107 \pm 3$ & - & $2.1 \pm 0.4$ & $0.20 \pm 0.05$ \\
\hline
\end{tabular}

${ }^{1}$ determined by Zetasizer with flat surface cell at $\mathrm{pH} 7,{ }^{2}$ determined from the distance of closest approach in water by SFA, ${ }^{3}$ Root Mean Square RMS: determined from AFM images $1 \mu \mathrm{m} \times 1 \mu \mathrm{m}$ scan size. Each experimental data represents the mean from a minimum of three measurements on two independent samples and the errors $( \pm)$ represent the standard deviation of the mean.

SAMs. Hydrophobized mica resulted in a hydrophobic smooth monolayer with water contact angle of $107^{\circ}$ and a thickness of $2.5 \mathrm{~nm}$ (Table 1). The positively charged aminofunctionalized mica substrate displayed also a smooth surface with a water contact angle of $78^{\circ}$, a surface zeta potential of $+40 \mathrm{mV}$ and a thickness of $2.5 \mathrm{~nm}$ (Table 1). The nonnull contact angle is associated with a small portion of the alkyl chain of aminoalkylsilane molecules which can be exposed to the aqueous surrounding environment, as previously demonstrated.[50] Hydrophobic monolayers have shown to remain stable in water over several days (Fig. S2) as it was previously reported for amino monolayers.[50]

\section{2 ss-siRNA interaction with the surfaces}

To establish a well-defined reference frame for the experiments, the forces between the bare and the functionalized substrates in water in absence of ss-siRNA were first measured. The reported force-distance profiles correspond to the first surface approach across Milli-Q water or ss-siRNA solution. The forces were also measured on separating 
the surfaces but are not reported as the system could be perturbed during the first compression and will not been representative of the non-perturbed ss-siRNA/substrate interactions. Briefly, adhesion was observed on separation for the three model surfaces and decreased after exposure to ss-siRNA (Fig. S3).

\section{Mica surface}

The force-distance profile measured between two bare mica surfaces across Milli-Q water clearly exhibits the long-range exponential decay that is characteristic of electrostatic double-layer interactions (Fig. 2.a). The surface potential ( $\left.\psi_{0}\right)$ was estimated to $90 \pm 22 \mathrm{mV}$ using Poisson-Boltzmann equation with a Debye length of $27 \pm 11 \mathrm{~nm}$. This value is in good agreement with the previously reported surface potential between mica surfaces across water.[51] At short separation distances, the attraction between mica surfaces is evidenced by a discontinuity in force profile from $5.0 \pm 0.4 \mathrm{~nm}$ to $\mathrm{D}=0 \mathrm{~nm}$ due to van der Waals attractive interactions (Fig. 2.a). Such discontinuity arises when the gradient of the force $(\mathrm{dF} / \mathrm{dD})$ exceeds the stiffness of the force measuring spring. The force-distance profile measured between mica surfaces across ss-siRNA solution is purely repulsive exhibiting also long-range electrostatic forces (Fig. 2.a). However, at smaller separation distances $(\mathrm{D}<5 \mathrm{~nm})$ the force deviates significantly, becoming increasingly repulsive upon decreasing separation distance. The additional repulsive force (compared to the forces in water) and the absence of discontinuity in the force profile indicate that the ss-siRNA is adsorbed to the mica surface. Attraction between oligonucleotides and mica in the presence of multivalent cations has already been theoretically predicted using counterion condensation theory.[52-54] However, to the best of our knowledge, this is the first experimental evidence of attractive interaction (or adhesion) between negatively charged mica surfaces and oligonucleotides in water. As ss-siRNA is expected to adsorb on both mica surfaces, the distance of closest approach (ca $3.3 \mathrm{~nm}$ ) is assumed to correspond to twice the thickness of immobilized oligonucleotides. The thickness of one adsorbed layer is close to the estimated radius of gyration $\left(\mathrm{R}_{\mathrm{g}}\right)$ of 19-bases RNA single strand in water $\left(\mathrm{R}_{\mathrm{g}}\right.$ $=1.5 \mathrm{~nm}$ ) [55] suggesting that adsorbed ss-siRNA forms a monolayer. The apparent surface potential $\psi_{0}$ at the ss-siRNA/water interface was estimated to $147 \pm 8 \mathrm{mV}$ using PoissonBoltzmann equation with a Debye length $\kappa^{-1}$ of $15.5 \pm 0.5 \mathrm{~nm}$ (Fig. 2.a). The relatively large 
surface potential, compared that of the bare mica substrate, can be attributed to the exposure of charged $\mathrm{PO}_{3}{ }^{3-}$ groups of RNA strands at the water/surface interface. Therefore, the interaction of ss-siRNA with mica most probably occurs through a combination of (i) attractive hydrogen bonding between the nucleobases and ribose of the ss-siRNA and the

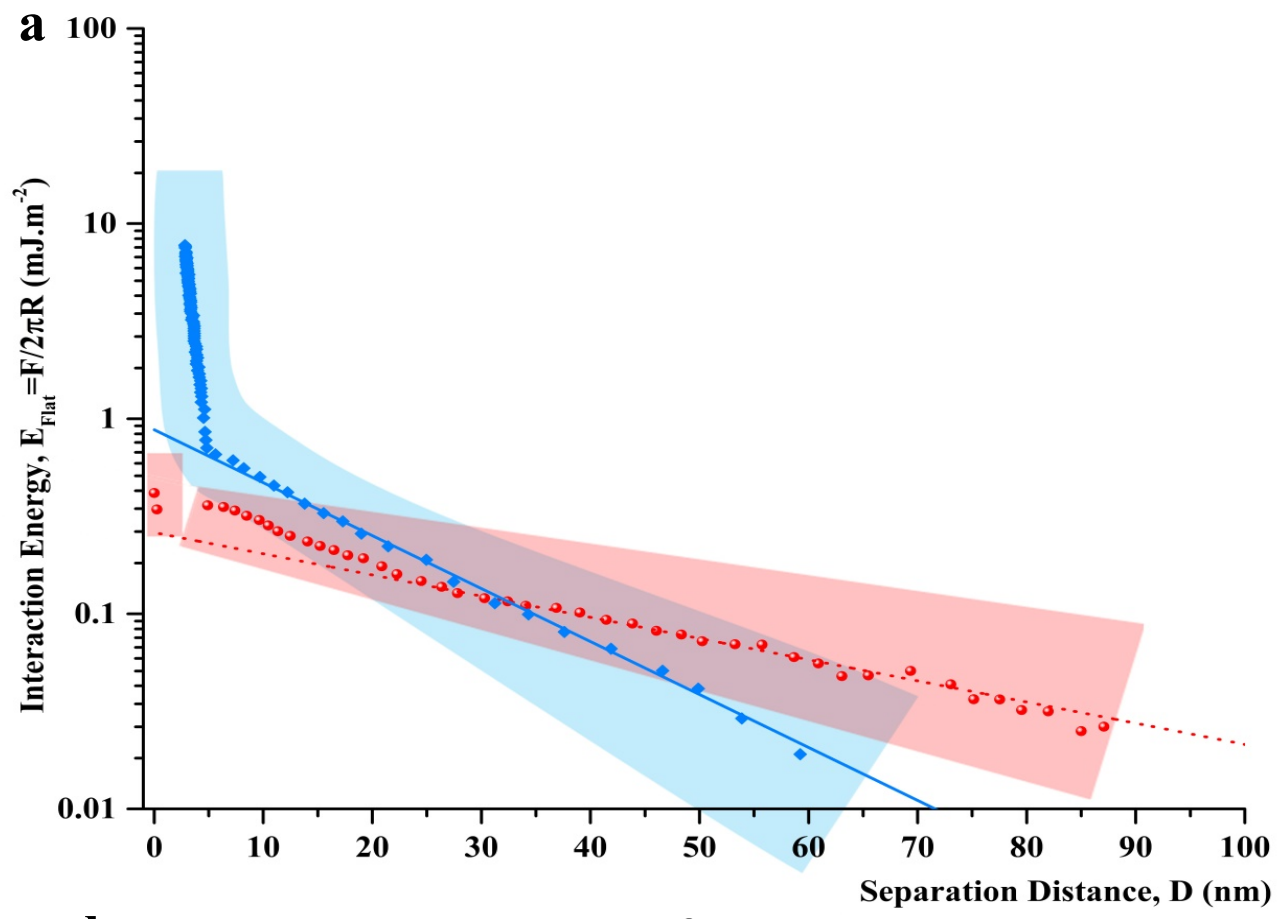

b
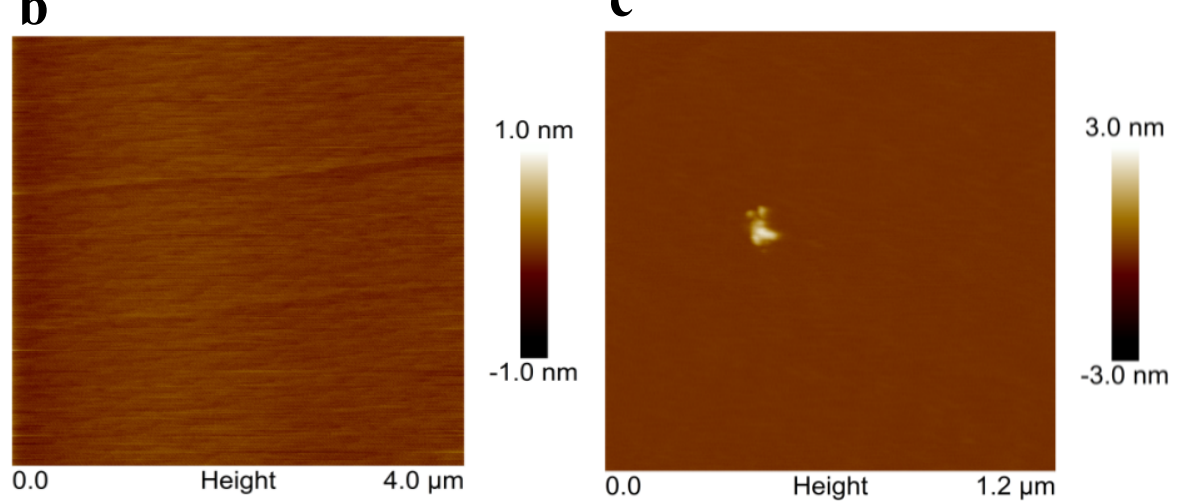

Figure 2: (a) Normalized force profiles, measured on the first surfaces approach, between two mica substrates across Milli-Q water $(\bullet)$ and across the ss-siRNA solution after two hours of immersion ( $\diamond$ ). $\mathrm{D}=0$ corresponds to the contact between mica surfaces in air. The dotted and solid curves correspond to the calculated electrostatic interactions using the Poisson-Boltzmann (PB) equation with a surface potential $\psi_{0}$ of $-115 \mathrm{mV}(\ldots)$ and $-155 \mathrm{mV}(-)$ and a Debye length $\kappa^{-1}$ of $40 \mathrm{~nm}(\ldots)$ and $16 \mathrm{~nm}(-)$ at room temperature. The shaded areas represent the variability in the measured force profiles and the most representative profile is illustrated. AFM images in air of (b) mica (c) mica after 20 hours of immersion in the ss-siRNA solution following by water rinsing. 
$\mathrm{SiO}^{-} / \mathrm{SiOH}$ groups of mica and (ii) attractive electrostatic interactions between the expected charged amino groups of nucleobases [56] and the $\mathrm{SiO}^{-}$groups of mica. The presence of hydrogen bonding between ss-DNA and an uncharged hydrophilic surface (composed of hydroxyl-terminated oligoethyleneglycol) has been evidenced by a simulation study.[36] Such interactions would suggest that ss-siRNA interacts with mica surface through the opposite nucleobases face as illustrated in Figure 1.c. However, the adsorbed ss-siRNA was easily removed from mica by water rinsing as the substrates recovered the bare mica topography (Fig. 2.c). This suggests that the electrostatic repulsion is sufficient to overcome the attraction between the negatively charged mica and the negatively charged ss-siRNA.

\section{Hydrophobized-mica surface}

The force-distance profile measured between two hydrophobized mica surfaces (prepared from dodecyltrichlorosilane) across Milli-Q water is purely attractive (Fig. 3.a). The longrange attraction is evidenced by a discontinuity in force profile from $3000 \mathrm{~nm}$ to $\mathrm{D}=4.0 \pm$ $0.4 \mathrm{~nm}$. This long-range interaction is characteristic of hydrophobic interactions across polar media.[57-59] The distance of closest approach $(4 \mathrm{~nm})$ corresponds to twice the thickness of one hydrophobic monolayer (Fig. 3.a). The force-distance profile between hydrophobized surfaces across the ss-siRNA solution is purely repulsive and well described by the electrostatic double-layer forces shown by the solid line fitted to the data points in Figure 3.a. In addition, the force deviates from the double-layer repulsions at relatively large separation distances ( $\mathrm{D}<30 \mathrm{~nm}$ ) and becomes significantly repulsive upon decreasing separation distance. This additional repulsive force compared to the forces in water) and the presence of well-defined hard wall at ca $8 \mathrm{~nm}$ clearly indicate the presence of adsorbed ss-siRNA on the hydrophobized substrates. The distance of closest approach is close to twice the estimated radius of gyration of ss-siRNA in water [55] suggesting the adsorption of ss-siRNA into a monolayer under high compression. The adsorption of sssiRNA is also evidenced by a change in the surface topography upon ss-siRNA exposition, which persisted even after intensive water rinsing (Fig. 3.b and c). The effective surface potential $\psi_{0}$ estimated from the Poisson-Boltzmann equation $(133 \pm 8 \mathrm{mV})$ and can be 


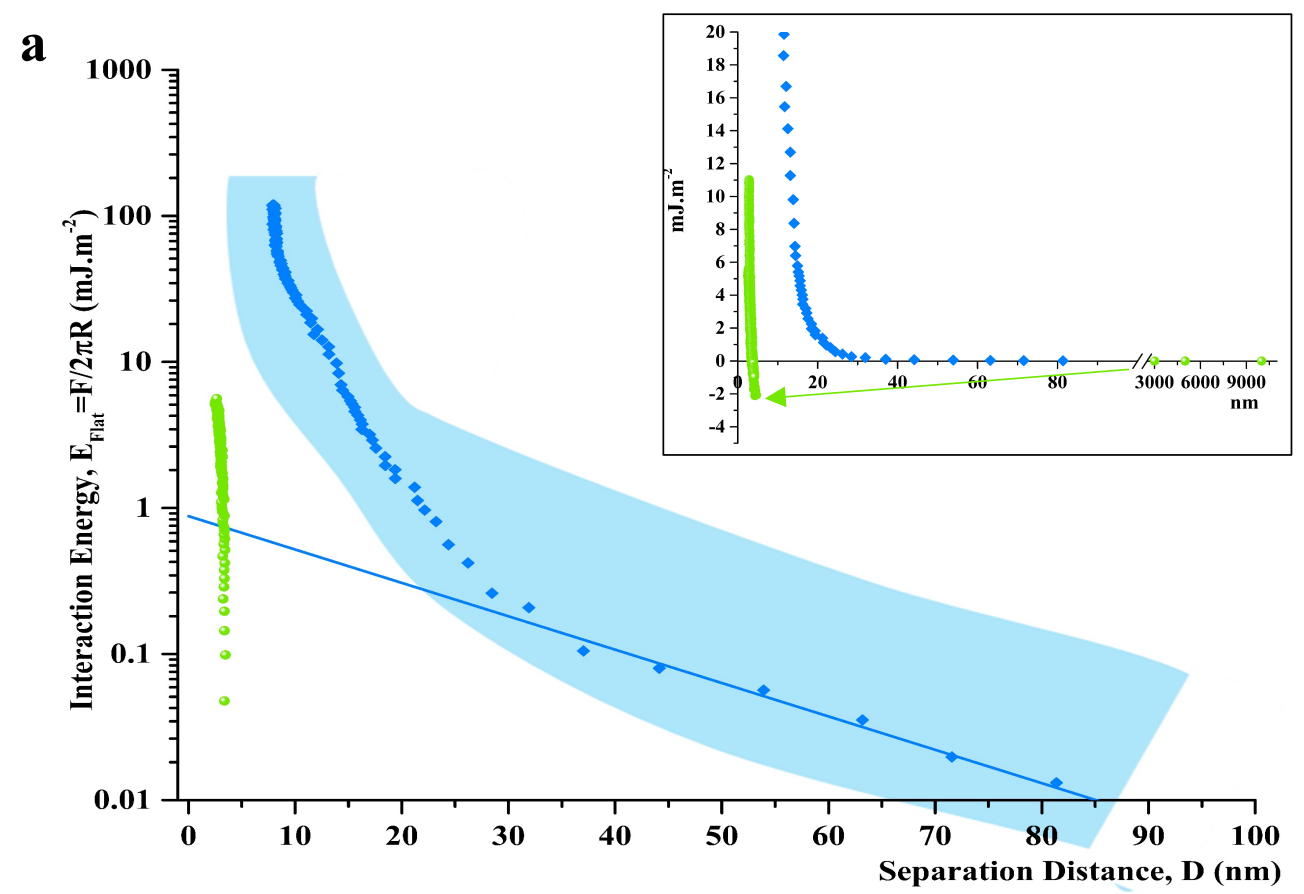

b
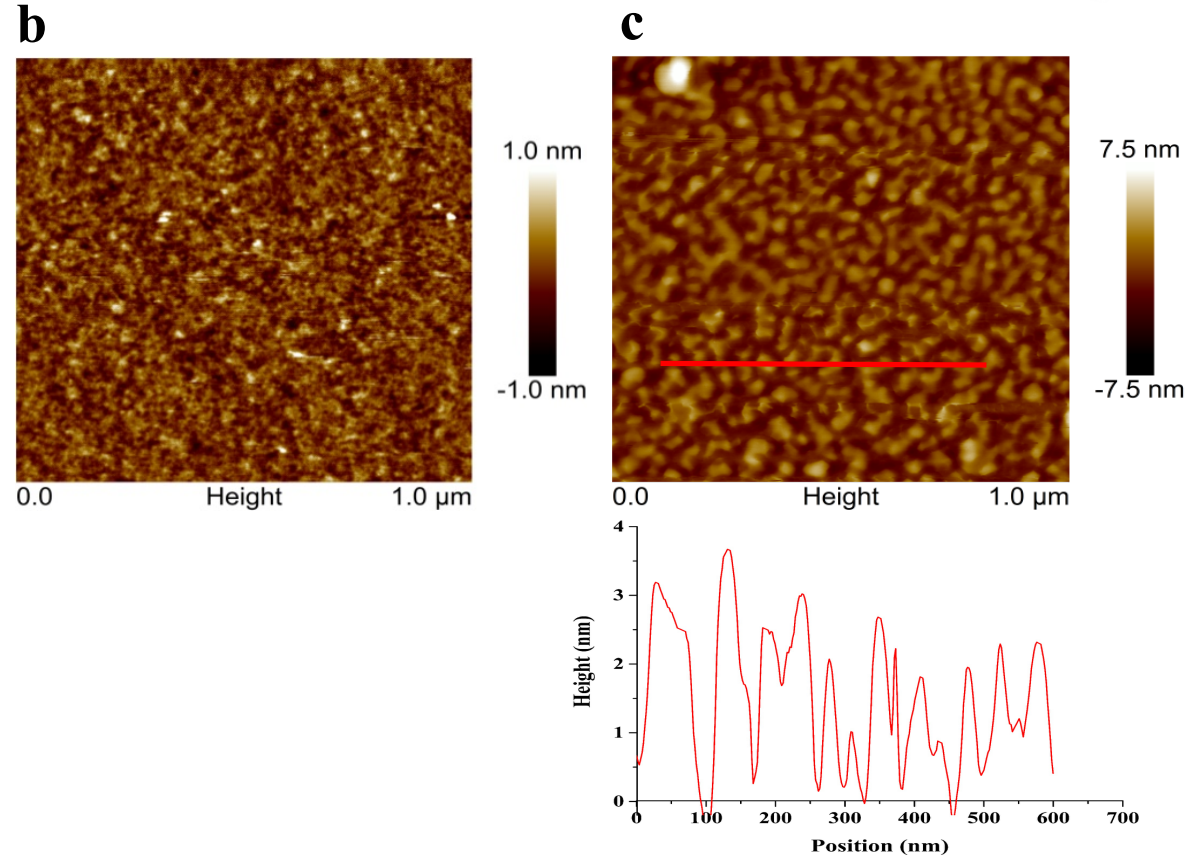

Figure 3: (a) Normalized force profiles, measured on the first approach, between two hydrophobized mica substrates across Milli-Q water $(\bullet)$ and across the ss-siRNA solution after two hours of immersion $(\diamond)$. The force profile between hydrophobized mica substrates across Milli-Q water, illustrated in the inset, is purely attractive as evidenced by a discontinuity in the force profile from $D=3 \mu \mathrm{m}$ to $\mathrm{D}=4 \mathrm{~nm}$ represented by the arrow. $\mathrm{D}=0$ corresponds to the contact between mica surfaces in air. The solid curve corresponds to the calculated electrostatic interactions using the Poisson-Boltzmann (PB) equation with a surface potential $\psi_{0}$ of $-140 \mathrm{mV}$ (-) and a Debye length $\kappa^{-1}$ of $19 \mathrm{~nm}(-)$ at room temperature. The shaded areas represent the variability in the measured force profiles and the most representative profiles are illustrated. AFM images in air of (b) hydrophobized mica and (c) hydrophobized mica after 2 hours of immersion in the ss-siRNA solution following by water rinsing with the corresponding cross section analysis. 
attributed to the exposure of the charged $\mathrm{PO}_{3}{ }^{3-}$ groups of RNA strands to the aqueous environment. Here again, these results suggest that the oligonucleotide interacts with hydrophobized mica surfaces through the opposite nucleobases face as illustrated in Figure 1.b. Hydrophobic interactions most probably occur through the alkyl chains of hydrophobic SAMs and the aromatics groups of the bases. These results correlate well with the previously reported simulation study showing attractive hydrophobic interactions between the strand face carrying nucleobases and a hydrophobic surface.[36]

\section{Amino-functionalized mica surface}

The force-distance profile measured between two amino-functionalized mica surfaces across Milli-Q water is illustrated in Figure 4.a. An effective positive surface potential was observed on these surfaces (Table 1) suggesting that the amino groups of the AUTES monolayers are protonated in Milli-Q water, as previously reported,[50] which should give rise to electrostatic interactions. However, such interactions were not detectable because of the important contribution of long-ranged attractive interactions (ca $300 \mathrm{~nm}$ ) most probably arising from hydrophobic interactions between the apposing alkyl chains of the two AUTES monolayers. At short separation distances $(<10 \mathrm{~nm})$, the attraction between the surfaces is evidenced by a discontinuity in force profile from $\mathrm{D}=10 \pm 1 \mathrm{~nm}$ to $5.0 \pm 0.5$ $\mathrm{nm}$ and is most probably due to van der Waals interactions (Fig. 4.a). The distance of the closest approach between amino-functionalized substrates is observed at separation distance of $3.0 \pm 0.2 \mathrm{~nm}$ (Fig. 4.a) corresponding to twice the thickness of an AUTES monolayer previously determined.[50] The force-distance profile between two aminofunctionalized mica surfaces across the ss-siRNA solution is purely repulsive but does not exhibit long-range exponential decaying forces (Fig. 4.a) suggesting the presence of steric forces associated with the adsorbed ss-siRNA. The distance of closest approach is shifted from $3.0 \pm 0.2 \mathrm{~nm}$ (in pure water) to a separation distance of $6.0 \pm 0.2 \mathrm{~nm}$ (Fig. 4.a). This shift corresponds to two ss-siRNA adsorbed layers of $1.5 \mathrm{~nm}$ each. The ss-siRNA adsorption was confirmed by the obvious change in the surface topography of the aminofunctionalized substrates observed after ss-siRNA exposition (Fig. 3. b and c). In addition, ss-siRNA remained adsorbed after water rinsing indicating the robustness of the adsorption (Fig. 3. c). The absence of long-range electrostatic repulsions between the ss-siRNA 


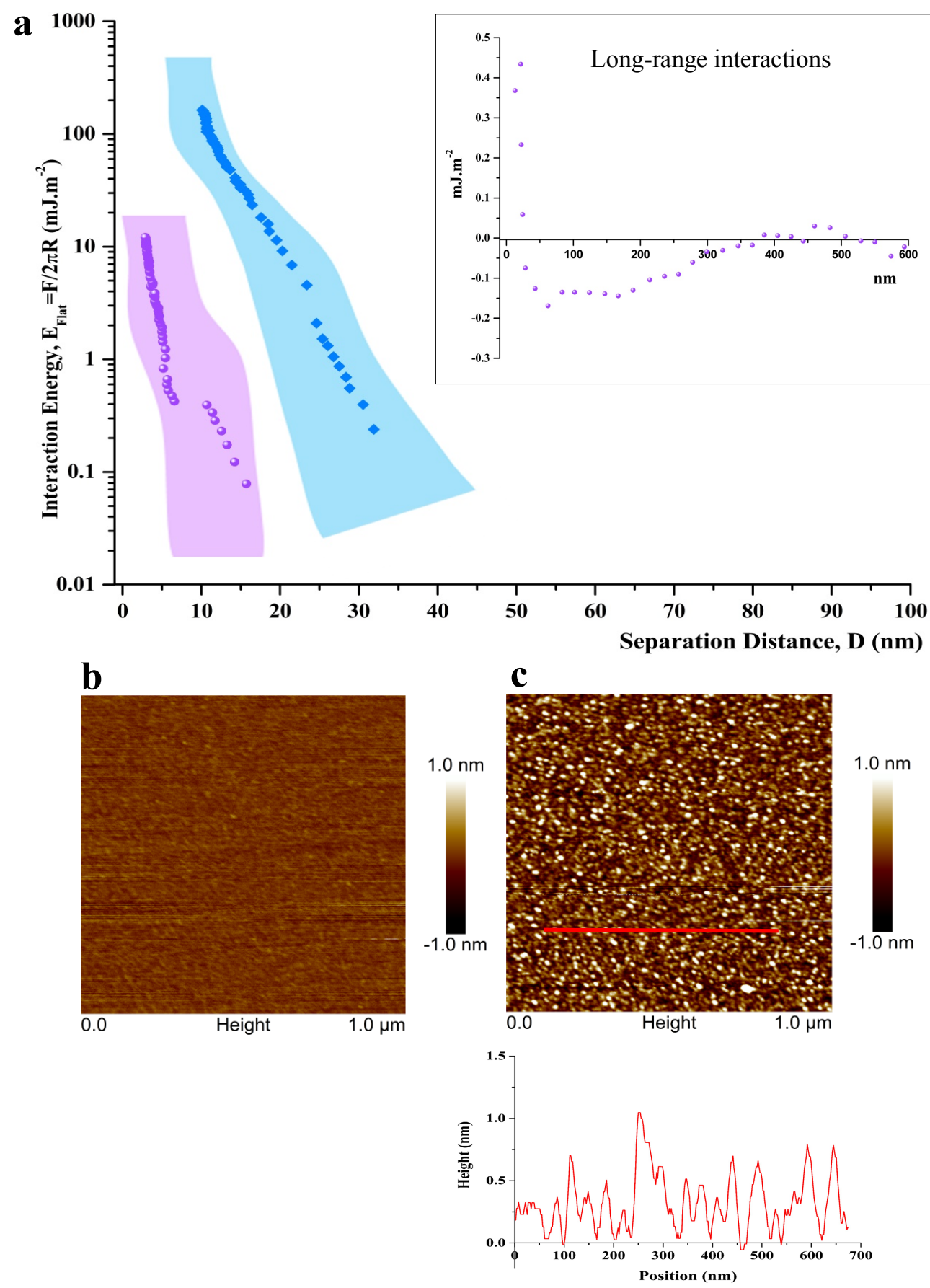

Figure 4: (a) Normalized force profiles, measured on the first surfaces approach, between two amino-functionalized mica substrates across Milli-Q water $(\bullet)$ and across the ss-siRNA solution after two hours of immersion ( $\diamond)$. Inset graphic is long-range interaction of normalized force profiles measured on the first approach between two amino-functionalized mica substrates across Milli-Q water. $\mathrm{D}=0$ corresponds to the contact between mica surfaces in air. The shaded areas represent the variability in the measured force profiles and the most representative profiles are illustrated. AFM images in air of (b) amino-functionalized mica and (c) amino-functionalized mica after 2 hours of immersion in the ss-siRNA solution following by water rinsing with the corresponding cross section analysis. 
covered amino-functionalized surfaces suggests a neutral ss-siRNA/water interface that could be associated with the exposition of the nucleobase face to the aqueous environment as illustrated in Figure 1.a. In such a conformation, ss-siRNA is likely adsorbed via electrostatic interactions between the positively charged amino groups of the SAMs and the negatively charged phosphate ribbon as previously reported for double strand DNA.[60] This would lead to a neutralization and/or screening of the charges across the adsorbed ss-siRNA layer on each surface.

\section{3 ss-siRNA interactions with DDS}

SFA experiments allowed the contribution of hydrogen bonding and hydrophobic interactions in the ss-siRNA adsorption, in addition to electrostatic forces, to be confirmed. To verify whether these interactions evidenced on planar surfaces could also be observed on more complex systems, such a drug carrier, and affect the loading capacity of DDS, liposomes and micelles of different compositions and surface charges were prepared, as illustrated in Figure 5. 1,2-dioleoyl-3-trimethylammonium-propane (chloride salt) (DOTAP) and 1,2-dimyristoyl-sn-glycero-3-phospho-(1-rac-glycerol) (sodium salt) (DMPG), 1-2-dioleoyl-sn-glycerol (DAG) were used to prepare cationic, anionic and uncharged liposomes respectively using cholesterol as co-lipid (Table S2). Zeta potential measurements confirmed their surface charge prior to ss-siRNA (Table 2). The unexpected negative potential measured for the DAG liposomes, most probably arises from the hydroxyl group of the hydrophilic head of DAG. Polymeric micelles were prepared using copolymers composed of a poly(styrene) (PS) hydrophobic block and a longer hydrophilic block of poly(N-methyl-4-vinyl pyridinium iodide) (PM4VPI), polyacrylic acid (PAA) and polyethyleneglycol) (PEG) to prepare cationic, anionic and uncharged micelles, respectively. Here again, the surface charges before ss-siRNA complexation were confirmed by zeta potential measurements (Table 2). Although incubation of ss-siRNA with DDS induced slight modifications of the diameter and charge of the DDS, all liposomal and micellar formulations loaded with ss-siRNA were stable at least over one day in Milli-Q water (Table 2).

ss-siRNA complexation was assessed indirectly by the interaction of unencapsulated ss-siRNA with the SYBR ${ }^{\circledR}$ Gold fluorescent dye. SYBR ${ }^{\circledR}$ Gold is an 
unsymmetrical cyanine dye positively charged due to the presence of tertiary amine groups that exhibits $>1000$-fold fluorescence enhancement upon intercalating to nucleic acids (double- or single-stranded DNA or to RNA).[42, 61-63] Fluorescence intensity is therefore directly correlated to the amount of accessible ss-siRNA in the solution, and is normalized by the fluorescence of a $250 \mathrm{nM}$ ss-siRNA solution with an excess of dye (Fig. 5)

Table 2: Characterization of DDS formulations before and after ss-siRNA complexation in Milli-Q water

\begin{tabular}{|c|c|c|c|c|c|c|}
\hline & \multicolumn{2}{|c|}{$\begin{array}{c}\text { Before } \\
\text { co-incubation }\end{array}$} & \multicolumn{2}{|c|}{$\begin{array}{l}\text { After } 30 \text { min of } \\
\text { co-incubation }\end{array}$} & \multicolumn{2}{|c|}{$\begin{array}{l}\text { After } 24 \text { h of } \\
\text { co-incubation }\end{array}$} \\
\hline & $\begin{array}{c}\text { Diameter } \\
(\mathbf{n m})\end{array}$ & $\begin{array}{c}\text { Zeta potential } \\
(\mathrm{mV}) \\
\end{array}$ & $\begin{array}{c}\text { Diameter } \\
(\mathbf{n m})\end{array}$ & $\begin{array}{c}\text { Zeta potential } \\
(\mathrm{mV}) \\
\end{array}$ & $\begin{array}{c}\text { Diameter } \\
(\mathbf{n m})\end{array}$ & $\begin{array}{c}\text { Zeta potential } \\
(\mathrm{mV})\end{array}$ \\
\hline \multicolumn{7}{|c|}{ Liposomal formulation } \\
\hline $\begin{array}{l}\text { Cationic } \\
\text { (DOTAP) }\end{array}$ & $263 \pm 3$ & $22 \pm 2$ & $113 \pm 1$ & $-20 \pm 1$ & $115 \pm 1$ & $-18 \pm 1$ \\
\hline $\begin{array}{l}\text { Anionic } \\
\text { (DMPG) }\end{array}$ & $212 \pm 8$ & $-30 \pm 1$ & $311 \pm 7$ & $-40 \pm 1$ & $302 \pm 3$ & $-40 \pm 1$ \\
\hline $\begin{array}{l}\text { Uncharged } \\
\text { (DAG) }\end{array}$ & $165 \pm 3$ & $-24 \pm 2$ & $238 \pm 1$ & $-43 \pm 2$ & $236 \pm 3$ & $-43 \pm 3$ \\
\hline \multicolumn{7}{|c|}{ Micellar formulation } \\
\hline $\begin{array}{c}\text { Cationic } \\
\text { (PS-b-PM4VPI) }\end{array}$ & $95 \pm 1$ & $58 \pm 1$ & $99 \pm 1$ & $48 \pm 2$ & $99 \pm 1$ & $50 \pm 2$ \\
\hline $\begin{array}{c}\text { Anionic } \\
\text { (PS-b-PAA) }\end{array}$ & $238 \pm 6$ & $-39 \pm 1$ & $170 \pm 3$ & $-38 \pm 2$ & $164 \pm 3$ & $-41 \pm 2$ \\
\hline $\begin{array}{l}\text { Uncharged } \\
\text { (PS-b-PEG) }\end{array}$ & $93 \pm 1$ & $-12 \pm 1$ & $96 \pm 1$ & $-12 \pm 1$ & $95 \pm 1$ & $-11 \pm 1$ \\
\hline
\end{tabular}

ss-siRNA/micelles complexations were done in Milli-Q water at room temperature for $14.3 \mu \mathrm{M}$ lipids, $11.9 \mu \mathrm{M}$ copolymers and $250 \mathrm{nM}$ ss-siRNA, respectively. Each experimental data represents the mean from a minimum of three measurements on one sample and the errors $( \pm)$ represent the standard deviation of the mean.

Unsurprisingly, cationic DDS were very efficient to complex ss-siRNA, reaching $95 \%$ and $100 \%$ of encapsulated material (Eq. 1) for cationic liposomes and micelles, respectively (Fig. 5.a and d). This is consistent with the widespread knowledge that an excess of cationic charge enables full complexation of oligonucleotides and promotes colloidal stability. The interaction most probably results from the complementary electrostatic nature of nucleic acid with cationic DDS as previously reported for double stranded siRNA complexation.[64] This is confirmed by the change in the size and the decrease of zeta potential for both systems (Table 2). However, cationic liposomal and 
Liposomal formulations

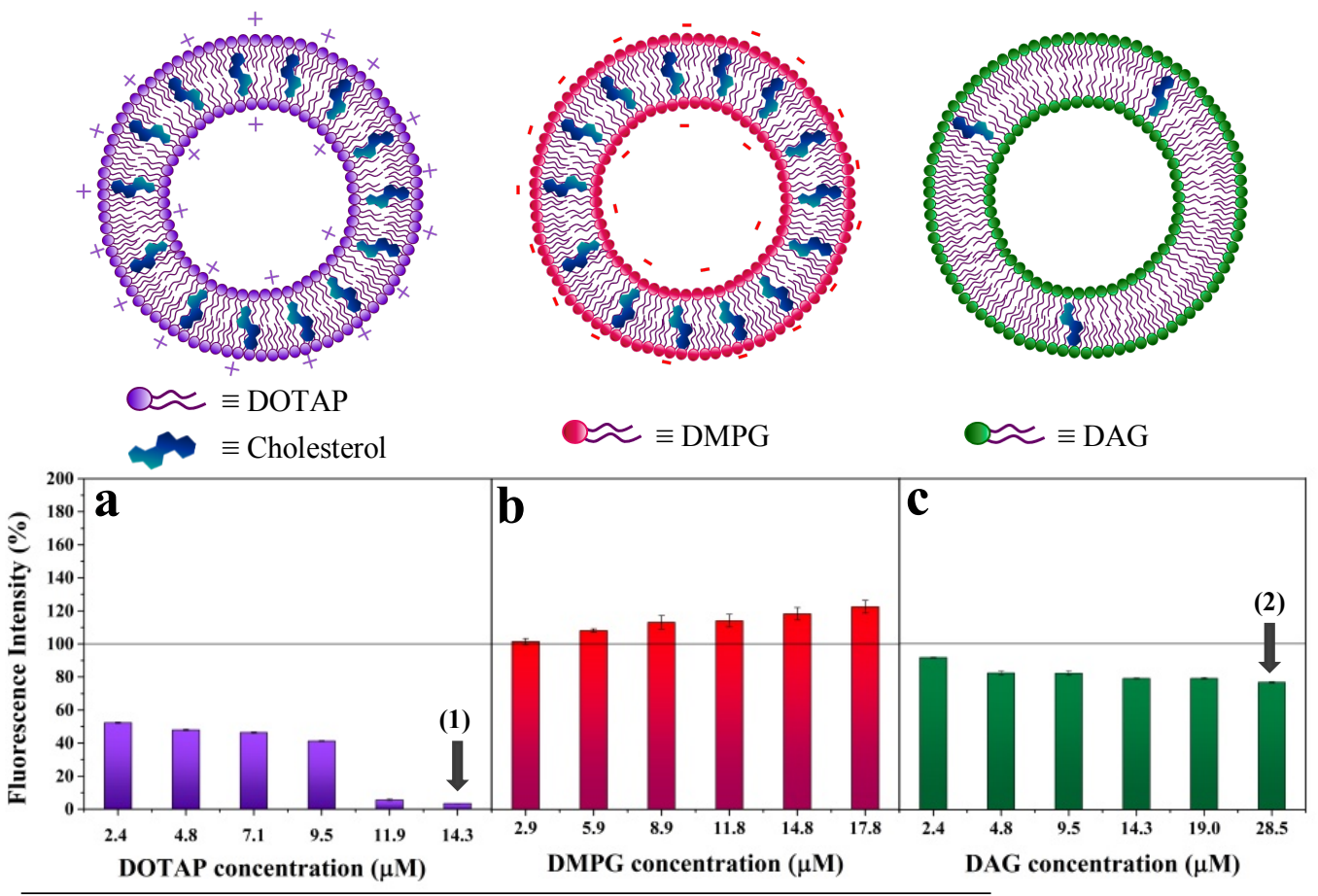

\section{Micellar formulations}

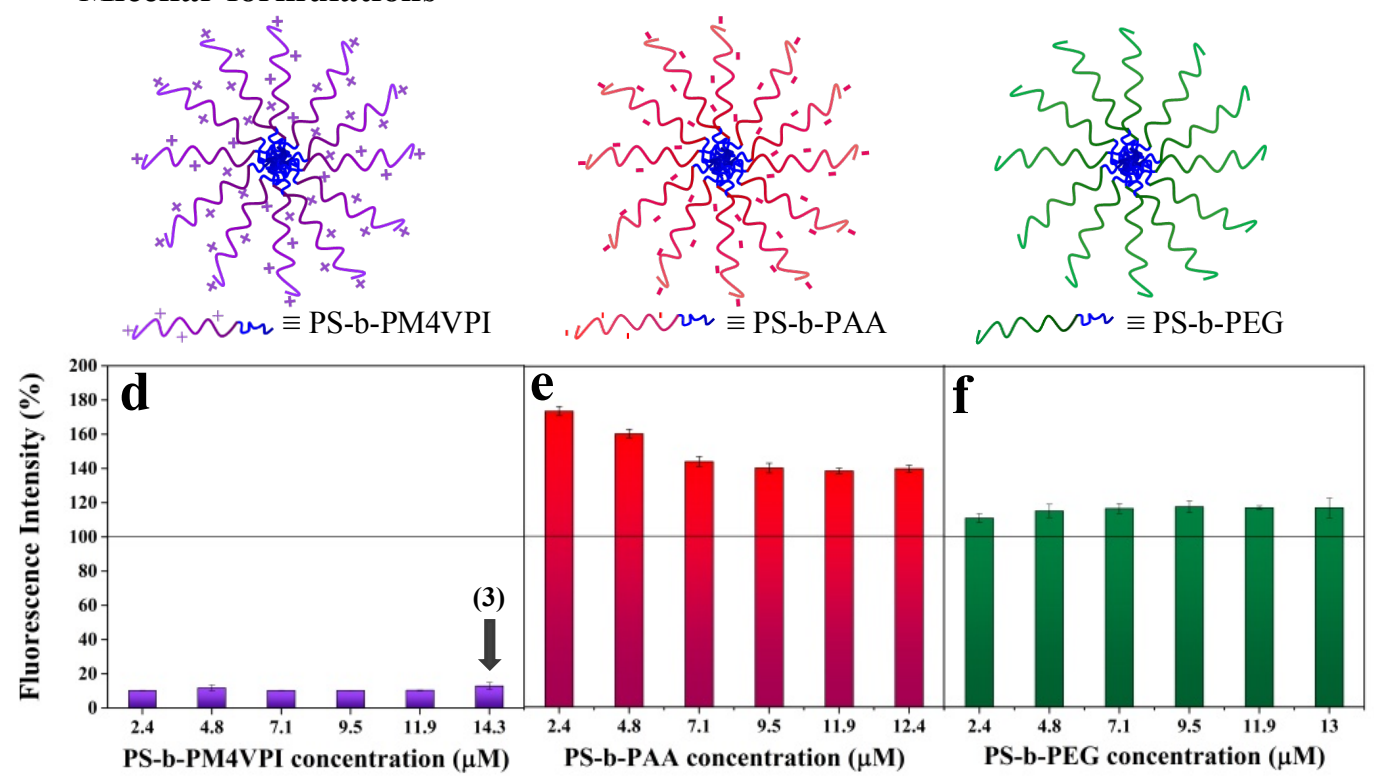

Figure 5: Normalized fluorescence intensity (\%) of the dye/ss-siRNA system in Milli-Q water with DDS as a function of concentration of (a) DOTAP, (b) DMPG and (c) DAG lipids for cationic, anionic and uncharged liposomes, respectively with their schematic representations and (d) PS-b-PM4VPI, (e) PS-b-PAA and (f) PS-b-PEG for cationic, anionic and uncharged micelles, respectively with their schematic representations. Fluorescence intensities were recorded at $537 \mathrm{~nm}$ and normalized to that of the dye/ss-siRNA system in Milli-Q for sssiRNA concentration of $250 \mathrm{nM}$ without DDS corresponding to $100 \%$ (indicated by horizontal dashed lines). Black arrows (1), (2) and (3) correspond to ss-siRNA encapsulation efficiency of $95 \%, 20 \%$ and $100 \%$, respectively. 
micellar formulations did not behave the same upon complexation of ss-siRNA, suggesting different complexation mechanisms. The size of cationic liposomes decreased when incubated with ss-siRNA whereas cationic micelles diameter slightly increased (Table 2). In addition, the surface potential of cationic micelles slightly decreased (Table 2) whereas that of the liposomes switched to negative value (Table 2). This difference in the surface potential changes is attributed to the significant difference in the charge excess between cationic liposomes (nitrogen/phosphorus ratio, N/P: 1.5) and micelles (N/P: 100). In fact, the surface charge of cationic micelles/ss-siRNA complex decreases with N/P ratio and becomes negative for N/P lower than 1.5 (Fig. S4).

With the uncharged liposomes, the normalized fluorescence intensity decreased with increasing lipid concentration suggesting a maximum encapsulation efficiency of $20 \%$ (Fig. 5.c). ss-siRNA association with uncharged liposomes was confirmed by the significant swelling of the liposomes and their higher negative character $(-43 \mathrm{mV}$, Table 2). Surprisingly, the behaviour was significantly different for uncharged micelles. Indeed, for the uncharged micelles as well as the anionic DDS (both liposomes and micelles), the fluorescence intensity exceeds $100 \%$ (Fig. 5.b, e and f) indicating that the fluorescence quantum yield of the dye/ss-siRNA system is larger than that of the reference system. It has to be noted that all samples were centrifuged after incubation with ss-siRNA, in order to sediment DDS. However, neither aggregation nor precipitation was evidenced even after extended period of centrifugation ( $2 \mathrm{~h}$ at $20000 \mathrm{~g}$, data not shown) for all liposome and micelle formulations. Therefore, ss-siRNA/DDS complexes can be present in the solution biasing the fluorescence measurements. As a control, no fluorescence of ss-siRNA/DDS or dye/DDS mixtures was detected for all DDS as presented for the anionic liposomes (Fig. S5. $\mathrm{b}$ and $\mathrm{c}$ ). We further investigated this unexpected phenomenon by recording the full emission spectrum of the three mixtures (Fig. 6). Interestingly, compared to the reference, the maximum of fluorescence emission spectra of the dye/ss-siRNA/anionic DDS mixture is clearly shifted to red and blue wavelengths for anionic liposomes and anionic micelles, respectively (Fig. 6.a and b) suggesting that the nucleotide is localized close to polar and less polar environment, respectively. The increase in quantum yield and the shift in the maximum wavelength evidenced a close interaction between the three partners (dye, sssiRNA and anionic DDS). The control experiments (Fig. S5) suggest that the three partners 
are required to interact closely, resulting in a higher quantum yield of the dye/sssiRNA/DDS system than the dye/ss-siRNA. Since SYBR ${ }^{\circledR}$ Gold possesses tertiary amines, its interaction with negatively charged ss-siRNA and anionic DDS could be enhanced by electrostatic interactions.
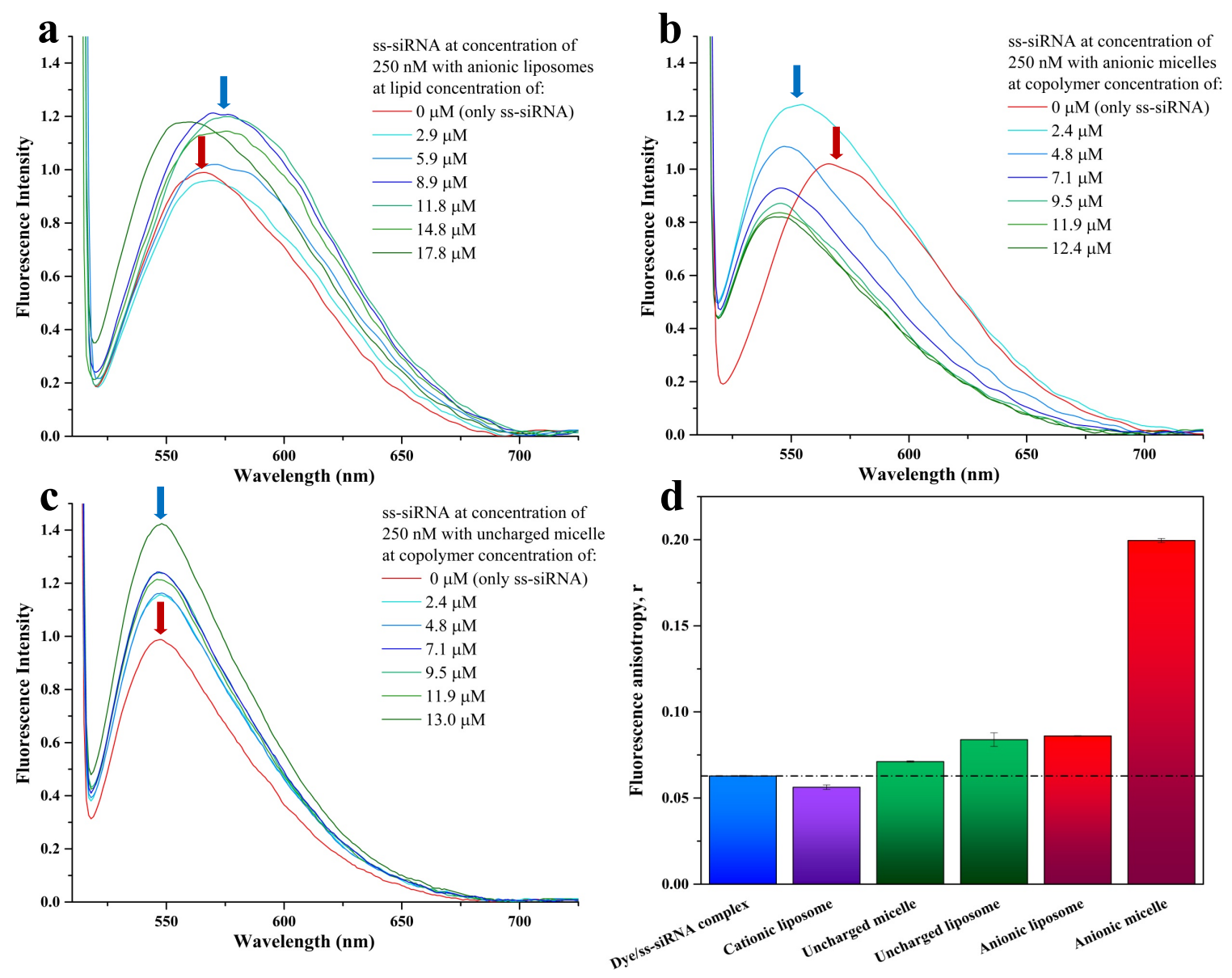

Figure 6: Normalized fluorescence emission spectra resulting from dye/ss-siRNA system in MilliQ water in the presence of (a) anionic liposomes, (b) anionic micelles and (c) uncharged micelles. Fluorescence intensities were normalized to that of the dye/ss-siRNA system in Milli-Q for sssiRNA concentration of $250 \mathrm{nM}$ without DDS. (d) Fluorescence anisotropy (r) of the dye/sssiRNA system without and with DDS with a lipid and copolymer concentrations of $11.9 \mu \mathrm{M}$ and a ss-siRNA concentration of $250 \mathrm{nM}$. Dashed-dot line indicated the fluorescence anisotropy resulting of the free dye/ss-siRNA system for a ss-siRNA concentration of $250 \mathrm{nM}$. Fluorescence anisotropy (r) of the dye/ss-siRNA system with cationic micelles is not reported as any significant fluorescence intensity can be measured as presented in Figure 5.d.

To confirm that the dye and the ss-siRNA are complexed together with the DDS, the fluorescence anisotropy was measured. This technique has been used to monitor the behaviour of lipid membranes and self-assemblies. It is sensitive to the motion of the probe 
itself as well as the motion of whole self-assemblies when the probe is associated with them.[65, 66] The fluorescence anisotropy of ss-siRNA complexed to SYBR ${ }^{\circledR}$ Gold was compared with and without the presence of the different DDS (Fig. 6.d). A small value of anisotropy (referred to r, see Eq. 2) indicates a low degree of polarization, and therefore a less rigid environment of the probe.[66] The dye/ss-siRNA exhibited a $r$ value of 0.063 in the absence of DDS (Fig. 6.d), indicating a high mobility of the ss-siRNA in solution. In the presence of cationic and anionic liposomes and neutral micelles, the $\mathrm{r}$ values were close to this reference value suggesting that the fluorescence emission mainly comes from the remaining dye/ss-siRNA complex localized outside of the DDS. However, with the uncharged liposomes as well as anionic DDS (both micelles and liposomes), significantly larger $r$ values were observed (Fig. 6.d), reaching 0.20 for anionic micelles. In this case, the ss-siRNA is accessible to the $\mathrm{SYBR}^{\circledR}$ Gold and the complex exhibited a lower mobility, suggesting that the dye interacted with ss-siRNA already complexed to the DDS. These results confirm that the fluorescent emission of the dye/ss-siRNA system does not result only from non-complexed ss-siRNA but also from the ss-siRNA complexed with DDS. Therefore, the change in fluorescence quantum yield of the dye/ss-siRNA system immobilized in DDS does not allow accurate quantification of the encapsulation. In particular, the complexation efficiency of uncharged liposomes (ca; $20 \%$, Fig. 5.c) was most probably underestimated as the measured fluorescence can result from encapsulated and free dye/ss-siRNA system.

The fluorescence anisotropy of the dye/ss-siRNA system can also provide information about the localization of the ss-siRNA in the DDS. The hydrophobic core of a micelle is known to be more rigid than its hydrophilic shell,[66] and could contribute to the large $r$ values observed with the anionic micelles, in addition to increasing amounts of complexed dye/ss-siRNA/DDS. In addition, the blue shift of the maximum of fluorescence emission of the dye/ss-siRNA/anionic micelle system indicates a less polar environment of the dye (Fig. 6.b). Therefore, fluorescence data clearly indicate that the ss-siRNA is localized inside the hydrophobic core. The variation in zeta potential of the anionic micelles upon ss-siRNA encapsulation (Table 2) supports that observation. The fluorescence anisotropy is relatively less pronounced for the other DDS and therefore, it is not possible to use such data to conclude about the localization of the nucleotide in the DDS. 
The complexation of ss-siRNA with anionic liposomes (DMPG) and with anionic micelles leads to an increase and a decrease in the formulation size, respectively (Table 2). This observation suggests that the nature of the interactions and the localization of the nucleotide are different for both liposome and micelle formulation. In the case of liposomes, the increase in the formulation size is most probably due to a change in the bilayer curvature caused by the partial insertion of the nucleotide and an increase in the membrane rigidity. This would suggest that interaction with the headgroups of the lipids, such as hydrogen bonding and electrostatic interactions between the positively charged nucleobases and the negatively charged lipids prevail. The decrease in the size of the anionic micelles results from the loading of the nucleotide into the hydrophobic core as confirmed by the fluorescence anisotropy results. A size reduction of micelles is usually explained by a relative reduction of the hydrophobic volume forming the core or an increase in the effective area of surfactant head groups of the micelle. As fluorescence anisotropy measurements showed that the nucleotide was preferentially localized into the micelle core, the decrease in the core size could be explained by enhanced hydrophobic interactions in presence of the nucleotide and therefore a shrinkage of the hydrophobic core.

For the cationic micelles, the ss-siRNA complexation observed with the SYBR ${ }^{\circledR}$ Gold assay together with the slight decrease in zeta potential of cationic micelles (Table 2) suggests that ss-siRNA interacts with the cationic shell. For the cationic liposomal formulations, the systematic decrease in zeta potential upon ss-siRNA complexation (Table 2) also suggests that ss-siRNA is also mainly localized at the surface of the lipid bilayer interacting with the hydrophilic head groups of the lipids. Interactions between ss-siRNA and the cationic DDS most probably occur through electrostatic interactions between the negatively charged ss-siRNA and the positively charged headgroups of the DDS as usually observed with cationic carriers.[23, 28, 67] The electrostatic interactions between sssiRNA and DOTAP cause the collapse of the liposomal formulation as evidenced by a decrease in the formulation size (Table 2). This collapse may arise from an increase in the effective area of the hydrophilic head groups in the outermost membrane layer due to the complexation with the ss-siRNA. Such packing change would result in a change in the membrane curvature that favors smaller size. With cationic micelles, slight changes in size 
were observed after ss-siRNA complexation for large N/P ratio (Table 2). This can be attributed to a small charge neutralization after ss-siRNA complexation as evidenced by slight decrease in zeta potential (Table 2). However, when the quantity of complexed sssiRNA increases, such as observed for lower N/P, the micelle/ss-siRNA size increases while the zeta potential decreases and become negative for $\mathrm{N} / \mathrm{P}=0.15$ (Table S4). This confirms that larger quantity of complexed ss-siRNA tends to increase the charge neutralization reducing the effective area of the headgroups and therefore, increasing the self-assembled structure size.

In summary, the combined data from fluorescence, zeta potential, size and force measurements clearly demonstrate the ability of anionic DDS to complex the nucleotide via different interactions. The hydrophobic interactions seem to prevail for the complexation of ss-siRNA with anionic micelles whereas hydrogen bonding and/or electrostatic interactions seem to dominate over the hydrophobic interaction for the complexation with anionic liposomes. On the other hand, the lack of changes in fluorescence properties (maximum of emission and anisotropy) or size and zeta potential (Table 2) of uncharged micelles upon ss-siRNA incubation clearly confirm the inability of PEG shell to complex ss-siRNA. The complexation of ss-siRNA with DAG liposomes most probably occurs through hydrogen bonding. Preferential interaction with the hydrophilic headgroup is evidenced by decrease in zeta potential upon ss-siRNA complexation (Table 2). In the absence of net charge in the hydrophilic headgroup, the interaction with ss-siRNA is expected to occur mainly through hydrogen bonding.

\section{Conclusion}

This study demonstrates for the first time that ss-siRNA can complex through nonelectrostatic interactions onto hydrophobic and negatively charged surfaces. This finding was first evidenced by measuring the adsorption of ss-siRNA layer onto model substrates using SFA. Moreover, the surface forces measurement demonstrated that the conformation depends on the nature of the interactions between the ss-siRNA and the surfaces. The complexation of ss-siRNA through non-electrostatic interactions was further evidenced with DDS such as micelles and liposomes using SYBR ${ }^{\circledR}$ Gold cyanine dye. Contrary to cationic DDS, the quantification of the ss-siRNA encapsulation efficiency can be biased 
with non-cationic DDS as the dye can interact with ss-siRNA already complexed to nanovectors. However, changes in fluorescence properties (amplitude and shift of the maximum of emission and anisotropy), size and zeta potential highlighted the ability of anionic DDS and uncharged liposomes to load ss-siRNA using non-electrostatic interactions. This fundamental investigation opens up new possibilities for designing DDS with controlled interactions for enhancing the efficiency in ss-siRNA complexation and its controlled release.

\section{Acknowledgements}

Financial support from the Natural Sciences and Engineering Research Council of Canada, Fonds Québécois pour la Recherche en Nature et Technologies, Groupe de Recherche Universitaire sur le Médicament, Centre de Recherche sur les Matériaux Auto-Assemblés and Canadian Society for Chemistry are acknowledged. Professor Michel Lafleur is also thanked for providing great discussion about fluorescence anisotropy results.

\section{Supplementary data}

Hydrolytic stability of hydrophobized-mica; SFA measurements on separation between models surfaces across water and a solution of ss-siRNA; fluorescence emission spectra of control experiments; HPLC methods used for the quantification of incorporated lipid into liposomes and the resulting incorporation.

\section{References}

[1] E. Bernstein, A.A. Caudy, S.M. Hammond, G.J. Hannon, Role of bidentate ribonuclease in the initiation of step of RNA interference Nature 409 (2001) 363-366.

[2] A. Fire, S. Xu, M.K. Montgomery, S.A. Kostas, S.E. Driver, C.C. Mello, Potent and specific genetic interference by double-stranded RNA in Caenorhabditis elegans, Nature, 391 (1998) 806811.

[3] S.M. Elbashir, J. Harborth, W. Lendeckel, A. Yalcin, K. Weber, T. Tuschl, Duplexes of 21nucleotide RNAs mediate RNA interference in cultured mammalian cells, Nature, 411 (2001) 494498.

[4] S. Crunkhorn, Success in Amyloidosis Trials Supports Potential of Systemic RNAi, Nat Rev Drug Discov., 12 (2013) 818-818.

[5] J. Li, X. Yu, Y. Wang, Y. Yuan, H. Xiao, D. Cheng, X. Shuai, A Reduction and pH DualSensitive Polymeric Vector for Long-Circulating and Tumor-Targeted siRNA Delivery, Adv. Mater, 26 (2014) 8217-8224. 
[6] E.P. Thi, C.E. Mire, A.C.H. Lee, J.B. Geisbert, J.Z. Zhou, K.N. Agans, N.M. Snead, D.J. Deer, T.R. Barnard, K.A. Fenton, I. MacLachlan, T.W. Geisbert, Lipid Nanoparticle siRNA Treatment of Ebola-virus-Makona-infected Nonhuman Primates, Nature 521 (2015) 362-365.

[7] R. Kole, A.R. Krainer, S. Altman, RNA therapeutics: beyond RNA interference and antisense oligonucleotides, Nat Rev Drug Discov, 11 (2012) 125-140.

[8] B.L. Davidson, A.M. Monteys, Singles Engage the RNA Interference Pathway, Cell, 150 (2012) 873-875.

[9] T. Holen, M. Amarzguioui, E. Babaie, H. Prydz, Similar behaviour of single-strand and doublestrand siRNAs suggests they act through a common RNAi pathway, Nucleic Acids Res., 31 (2003) 2401-2407.

[10] W.F. Lima, T.P. Prakash, H.M. Murray, G.A. Kinberger, W. Li, A.E. Chappell, C. S., S.F. Murray, H. Gaus, P.P. Seth, E.E. Swayze, S.T. Crooke, Single-Stranded siRNAs Activate RNAi in Animals, Cell, 150 (2012) 883-894.

[11] A.L. Jackson, P.S. Linsley, Recognizing and avoiding siRNA off-target effects for target identification and therapeutic application, Nat. Rev. Drug Discov., 9 (2010) 57-67.

[12] Y. Fedrov, E.M. Anderson, A. Birmingham, A. Reynolds, J. Karpilow, K. Robinson, D. Leake, W.S. Marshall, A. Khvorova, Off-target effects by siRNA can induce toxic phenotype, RNA, 12 (2006) 1188-1196.

[13] A.L. Jackson, S.R. Bartz, J. Schelter, S.V. Kobayashi, J. Burchard, M. Mao, B. Li, G. Cavet, P.S. Linsley, Expression profiling reveals off-target gene regulation by RNAi, Nat. Biotechnol., 21 (2003) 635-637.

[14] J.B. Bramsen, M.B. Laursen, C.K. Damgaard, S.W. Lena, B.R. Babu, J. Wengel, J. Kjems, Improved silencing properties using small internally segmented interfering RNAs, Nucleic Acids Res., 35 (2007) 5886-5897.

[15] N. Dias, C.A. Stein, Antisense Oligonucleotides: Basic Concepts and Mechanisms, Mol Cancer Ther, 1 (2002) 347-355.

[16] Y. Xia, J. Tian, X. Chen, Effect of surface properties on liposomal siRNA delivery, Biomaterials, 79 (2016) 56-68.

[17] K.A. Whitehead, R. Langer, D.G. Anderson, Knocking down barriers: advances in siRNA delivery, Nat. Rev. Drug Discov., 8 (2009) 129-138.

[18] R. Kanasty, J.R. Dorkin, A. Vegas, D. Anderson, Delivery materials for siRNA therapeutics, Nat. Mat 12 (2013) 967-977.

[19] D.H. Kim, J.J. Rossi, Strategies for silencing human disease using RNA interference, Nature Rev. Genet., 8 (2007) 173-184.

[20] J.M. Layzer, A.P. McCaffrey, A. K.Tanner, Z. Huang, M.A. Kay, B.A. Sullenger, In vivo activity of nuclease-resistant siRNAs, RNA (2004), 10 (2004) 766-771.

[21] J. Wang, Z. Lu, M.G. Wientjes, J.L.-S. Au, Delivery of siRNA Therapeutics: Barriers and Carriers, AAPS J. , 12 (2010) 492-503.

[22] K.T. Love, K.P. Mahon, C.G. Levins, K.A. Whitehead, W. Querbes, J.R. Dorkin, J. Qin, W. Cantley, L.L. Qin, T. Racie, M. Frank-Kamenetsky, K.N. Yip, R. Alvarez, D.W.Y. Sah, A.d. Fougerolles, K. Fitzgerald, V. Koteliansky, A. Akinc, R. Langer, D.G. Anderson, Lipid-like materials for low-dose, in vivo gene silencing, PNAS, 107 (2010) 1864-1869.

[23] H.-K. Kim, E. Davaa, C.-S. Myung, J.-S. Park, Enhanced siRNA delivery using cationic liposomes with new polyarginine-conjugated PEG-lipid, Int. J. Pharm., 392 (2010) 141-147.

[24] D. Zhi, S. Zhang, S. Cui, Y. Zhao, Y. Wang, D. Zhao, The Headgroup Evolution of Cationic Lipids for Gene Delivery, Bioconjugate Chem, 24 (2013) 487-519.

[25] M.E. Davis, The First Targeted Delivery of siRNA in Humans via a Self-Assembling, Cyclodextrin Polymer-Based Nanoparticle: From Concept to Clinic, Mol. Pharm., 6 (2009) 659668.

[26] V.D. Badwaik, E. Aicart, Y.A. Mondjinou, M.A. Johnson, V.D. Bowman, D.H. Thompson, Structure-property relationship for in vitro siRNA delivery performance of cationic 2- 
hydroxypropyl-beta-cyclodextrin: PEG-PPG-PEG polyrotaxane vectors, Biomaterials, 84 (2016) 86-98.

[27] M.E. Davis, J.E. Zuckerman, C.H.J. Choi, D. Seligson, A. Tolcher, C.A. Alabi, Y. Yen, J.D. Heidel, A. Ribas, Evidence of RNAi in humans from systemically administered siRNA via targeted nanoparticles, Nature 464 (2010) 494-498.

[28] D.C. Forbes, N.A. Peppas, Polycationic nanoparticles for siRNA delivery: comparing ARGET ATRP and UV-initiated formulations, ACS Nano, 8 (2014) 2908-2917.

[29] J.E. Zuckerman, M.E. Davis, Clinical experiences with systemically administered siRNAbased therapeutics in cancer, Nat. Rev. Drug Discov., 14 (2015) 843-856.

[30] S.-Y. Lee, M.S. Huh, S. Lee, S.J. Lee, H. Chung, J.H. Park, Y.-K. Oh, K. Choi, K. Kim, I.C.

Kwon, Stability and cellular uptake of polymerized siRNA (poly-siRNA)/polyethylenimine (PEI) complexes for efficient gene silencing, J. Control. Release, 141 (2010) 339-346.

[31] H.J. Haringsma, J.J. Li, F. Soriano, Denise M. Kenski, W.M. Flanagan, A.T. Willingham, mRNA knockdown by single strand RNA is improved by chemical modifications, Nucleic Acids Res., 40 (2012) 4125-4136.

[32] A. Malek, O. Merkel, L. Fink, F. Czubayko, T. Kissel, A. Aigner, In vivo pharmacokinetics, tissue distribution and underlying mechanisms of various PEI(-PEG)/siRNA complexes, Toxicol. Appl. Pharmacol., 236 (2009) 97-108.

[33] F. Alexis, E. Pridgen, L.K. Molnar, O.C. Farokhzad, Factors Affecting the Clearance and Biodistribution of Polymeric Nanoparticles, Mol. Pharm., 5 (2008) 505-515.

[34] S.C. Semple, A. Akinc, J. Chen, A.P. Sandhu, B.L. Mui, C.K. Cho, D.W.Y. Sah, D. Stebbing, E.J. Crosley, E. Yaworski, I.M. Hafez, J.R. Dorkin, J. Qin, K. Lam, K.G. Rajeev, K.F. Wong, L.B. Jeffs, L. Nechev, M.L. Eisenhard, M. Jayaraman, M. Kazem, M.A. Maier, M. Srinivasulu, M.J. Weinstein, Q. Chen, R. Alvarez, S.A. Barros, S. De, S.K. Klimuk, T. Borland, V. Kosovrasti, W.L. Cantley, Y.K. Tam, M. Manoharan, M.A. Ciufolini, M.A. Tracy, A.d. Fougerolles, I. MacLachlan, P.R. Cullis, T.D. Madden, M.J. Hope, Rational design of cationic lipids for siRNA delivery, Nat. Biotechnol., 28 (2010) 172-176.

[35] S.T. Crowley, J.A. Poliskey, N.J. Baumhover, K.G. Rice, Efficient expression of stabilized mRNA PEG-peptide polyplexes in liver, Gene Ther, 22 (2015) 993-999.

[36] R.M. Elder, A. Jayaraman, Structure and thermodynamics of ssDNA oligomers near hydrophobic and hydrophilic surfaces, Soft Matter, 9 (2013) 11521-11533.

[37] M. Kastantin, D.K. Schwartz, DNA Hairpin Stabilization on a Hydrophobic Surface, Small, 9 (2013) 933-941.

[38] J.H. Monserud, D.K. Schwartz, Effects of Molecular Size and Surface Hydrophobicity on Oligonucleotide Interfacial Dynamics, Biomacromolecules, 13 (2012) 4002-4011.

[39] G. Wang, T. Zhao, L. Wang, B. Hu, A. Darabi, J. Lin, M.M.Q. Xing, X. Qiu, Studying Different Binding and Intracellular Delivery Efficiency of ssDNA Single-Walled Carbon Nanotubes and Their Effects on LC3- Related Autophagy in Renal Mesangial Cells via miRNA382, ACS Appl. Mater. Interfaces, 7 (2015) 25733-25740.

[40] V. Patzel, S. Rutz, I. Dietrich, C. Koberle, A. Scheffold, S.H. Kaufmann, Design of siRNAs producing unstructured guide-RNAs results in improved RNA interference efficiency, Nat Biotechnol, 23 (2005) 1440-1444.

[41] F. Xia, X. Zuo, R. Yang, Y. Xiao, D. Kang, A. Vallee-Belisle, X. Gong, A.J. Heeger, K.W. Plaxco, On the binding of cationic, water-soluble conjugated polymers to DNA: electrostatic and hydrophobic interactions, J Am Chem Soc, 132 (2010) 1252-1254.

[42] R.S. Tuma, M.P. Beaudet, X. Jin, L.J. Jones, C.-Y. Cheung, S. Yue, V.L. Singer, Characterization of SYBR Gold nucleic acid gel stain: a dye optimized for use with 300-nm ultraviolet Anal. Chem., 268 (1999) 278-288.

[43] M. Zheng, D. Librizzi, A. Kılıç, Y. Liu, H. Renz, O.M. Merkel, T. Kissel, Enhancing in vivo circulation and siRNA delivery with biodegradable polyethylenimine-graft-polycaprolactoneblock-poly(ethylene glycol) copolymers, Biomaterials, 33 (2012) 6551-6558. 
[44] T. Lobovkina, G.B. Jacobson, E.G. Gonzalez, R.P. Hickerson, D. Leake, R.L. Kaspar, C.H. Contag, R.N. Zare, In Vivo Sustained Release of siRNA from Solid Lipid Nanoparticles, ACS Nano, 5 (2011) 9977-9983.

[45] B. Liberelle, X. Banquy, S. Giasson, Stability of Silanols and Grafted Alkylsilane Monolayers on Plasma-Activated Mica Surfaces, Langmuir, 24 (2008) 3280-3288.

[46] Y. Yang, A.M. Bittner, S. Baldelli, K. Kern, Study of Self-Assembled Triethoxysilane Thin Films Made by Casting Neat Reagents in Ambient Atmosphere, Thin Solid Films, 516 (2008) 39483956.

[47] J. Israelachvili, Y. Min, M. Akbulut, A. Alig, G. Carver, WGreene, K. Kristiansen, E. Meyer, N. Pesika, K. Rosenberg, H. Zeng, Recent Advances in the Surface Forces Apparatus (SFA) Technique, Rep. Prog. Phys, 73 (2010) 1-16.

[48] J.N. Israelachvili, Thin Film Studing Using Multiple-Beam Interferometry, J. Colloid Interface Sci., 44 (1972) 259-272.

[49] B. Derjaguin, Untersuchungen über die Reibung und Adhäsion, IV, Kolloid-Z., 69 (1934) 155164.

[50] L. Giraud, R. Nadarajah, Y. Matar, G. Bazin, J. Sun, X.X. Zhu, S. Giasson, Aminofunctionalized Monolayers Covalently Grafted to Silica-Based Substrates as a Robust Primer Anchorage in Aqueous Media, Appl. Surf. Sci, 370C (2016) 476-485.

[51] S. Giasson, T.L. Kuhl, J.N. Israelachvili, Adsorption and Interaction Forces of Micellar and Microemulsion Solutions in Ultrathin Films, Langmuir, 14 (1998) 891-898.

[52] H.G. Hansma, D.E. Laney, DNA Binding to Mica Correlates with Cationic Radius: Assay by Atomic Force Microscopy, Biophysical Journal, 70 (1996) 1933-1939.

[53] Y. Kan, Q. Tan, G. Wu, W. Si, Y. Chen, Study of DNA adsorption on mica surfaces using a surface force apparatus, Scientifc Reports 5(2015) 1-6.

[54] J. Adamcik, D.V. Klinov, G. Witz, S.K. Sekatskii, G. Dietler, Observation of single-stranded DNA on mica and highly oriented pyrolytic graphite by atomic force microscopy, FEBS Letters, 580 (2006) 5671-5675.

[55] A. Werner, Predicting translational diffusion of evolutionary conserved RNA structures by the nucleotide number, Nucleic Acids Res., 39 (2011) e17.

[56] M. Blackburn, Nucleic acid in chemistry and biology 3rd edition, (2006) p16.

[57] F. Despa, R.S. Berry, The Origin of Long-Range Attraction between Hydrophobes in Water, Biophysical Journal, 92 (2007) 373-378.

[58] E.E. Meyer, K.J. Rosenberg, J. Israelachvili, Recent progress in understanding hydrophobic interactions, PNAS, 103 (2006) 15739-15746.

[59] S.H. Donaldson, Jr., S. Das, M.A. Gebbie, M. Rapp, L.C. Jones, Y. Roiter, P.H. Koenig, Y. Gizaw, J.N. Israelachvili, Asymmetric electrostatic and hydrophobic-hydrophilic interaction forces between mica surfaces and silicone polymer thin films, ACS Nano, 7 (2013) 10094-10104.

[60] L.S. Shlyakhtenko, A.A. Gall, Y.L. Lyubchenko, Mica Functionalization for Imaging of DNA and Protein-DNA Complexes with Atomic Force Microscopy, Methods Mol Biol. , 931 (2013) 295-312.

[61] S.T. Yue, V. L.Singer, B.L. Roth, T.J. Mozer, P.J. Millard, L.J. Jones, X. Jin, R.P. Haugland, U.S Patent No., 5,658,751 (1997).

[62] R.P. Haugland, S.T. Yue, P.J. Millard, B.L. Roth, U.S Patent No., 5,436,134 (1995).

[63] W.R. Strapps, V. Pickering, G.T. Muiru, J. Rice, S. Orsborn, B.A. Polisky, A. Sachs, S.R. Bartz, The siRNA sequence and guide strand overhangs are determinants of in vivo duration of silencing, Nucleic Acids Res., 38 (2010) 4788-4797.

[64] S. Xu, M. Dong, X. Liu, K.A. Howard, J. Kjems, F. Besenbacher, Direct Force Measurements between siRNA and Chitosan Molecules Using Force Spectroscopy, Biophysical Journal, 93 (2007) 952-959.

[65] H.M. Burt, J.K. Jackson, Monosodium Urate Monohydrate Crystal Induced Changes in Membrane Fluidity: a Fluorescence Polarization Study., Rheumatology, 15 (1988) 1144-1151. 
[66] X. Zhang, J.K. Jackson, H.M. Burt, Determination of Surfactant Critical Micelle Concentration by a Novel Fluorescence Depolarization Technique, Biochem. Biophys. Methods 31 (1996) 145150.

[67] Y. Sato, H. Hatakeyama, Y. Sakuraia, M. Hyodo, b. Hidetaka Akitaa, Hideyoshi Harashima, A pH-sensitive cationic lipid facilitates the delivery of liposomal siRNA and gene silencing activity in vitro and in vivo, J. Control. Release, 10 (2012) 267-276. 